\title{
An Energy Competent Steering Protocol using CSA to Increase Life of WSN
}

\author{
Parwinder Kaur \\ Department of Computer \\ Science Engineering \\ BMSCE, Muktsar, India
}

\author{
Gurjot Kaur \\ Department of Computer \\ Science Engineering \\ BMSCE, Muktsar, India
}

\begin{abstract}
These days proficient plan and realization of wireless sensor networks has turn into a wide spread area of study in current time, all because of the immense capability of sensor networks to permit applications that bond the substantial world to virtual world. Wireless sensor network (WSN) is being worn in surveillance, medical examination etc. Sensors nodes are typically built of few sensors and a mote unit. Sensor is a portion of equipment which wits the data and hands it over to mote. Sensors are usually worn to compute changes in substantial environmental parameters such as warmth, strain, moisture, noise, and tremor. Also variations in the fitness parameter of someone e.g. blood pressure and the rate at which the heart beats. In this research a technique named Energy competent Steering Protocol based on CSA (ECSPCSA) is projected to apply Cuckoo Search Algorithm (CSA) to the problem of electing the group leader $(\mathrm{CH})$ in the group set-up stage based on minimizing the intra group average space linking the member sensors and their relevant leaders $(\mathrm{CH})$ and minimizing the total of fraction of remaining power/energy of active sensors in group to energy intensity of leader $(\mathrm{CH})$ to proficiently increase the network life span and to get better stability period.
\end{abstract}

\section{Keywords}

WSN (Wireless Sensor Network), CSA (Cuckoo Search Algorithm).

\section{INTRODUCTION}

WSN is a self configuring network of tiny sensor nodes interacting amongst themselves via radio waves and positioned in number to intellect, watch and comprehend the substantial world. By grouping huge figures of small sensor nodes, it is likely to get figures about substantial event that was tricky or unfeasible to attain in more predictable behavior. In the approaching years, as progress in micro fabrication tools agree to the expenditure of developing sensor nodes to prolong to drop, growing consumptions of WSN's are predictable, with the networks ultimately increasing to hefty numbers of nodes. Probable applications for such largescale WSN's exist in a variety of fields, including medical examination [1, 2], environmental examination [3], supervision, habitat safekeeping, armed operations, and business instrument monitoring.

The paper trails as: Analysis of earlier work is specified in Section. II. Section III focuses on the formulation of the projected algorithm. Section IV hear say a number of tentative results to display the performance of the new algorithm. In the end, conclusions are drawn in Section. V.

\section{LITERATURE SURVEY}

Hoang et. al. (2014) proposed a structure that enables sensible progress of innermost cluster-based protocols reinforced by upsurge methods for the wireless sensor networks. Based on this structure, a protocol employing harmony search algorithm (HSA), was planned and realized in real world for the WSNs. From the tentative examination, it indicates that the WSNs life span has been increased via the planned HSA protocol in contrast to that of LEACH-C and FCM protocols [5].

Attea et. al. (2012) aimed to improve the unwanted activities of the EA when trading with clustered steering issue in WSN by creating a new fitness function that include 2 clustering aspects, viz. consistency and parting error. Imitation on 20 random varied WSNs shows that author's evolutionary based clustered routing protocol (ERP) at all times extends the network life span, conserves further energy in contrast to the outcome attained employing the current heuristics like LEACH, SEP, and HCR protocols. [6].

A. Zahmatkesh and M. H. Yaghmaee (2012) proposed Genetic Algorithm abbreviated as GA to reform sensor nodes' energy utilization. The authors used a multipurpose algorithm that produces most favorable number of sensor-clusters with cluster-heads and reduces the fee of broadcast. The apparatus are then utilized and the average fitness of the system is calculated [7].

Smaragdakis et. al. (2004) projected Stable Election Protocol (SEP), a heterogeneous-aware protocol to extend the life span before the first node is lost, which is vital for numerous applications where the reaction from the sensor network must be dependable. SEP is based on biased selection probabilities of each node to turn into cluster head as per the lingering energy in every node [8].

Heinzelman et. al. (2002) build up and examine low energy adaptive clustering hierarchy (LEACH), a protocol structural design for micro-sensor networks that unites the thoughts of energy efficient cluster based routing and media access jointly along with application-specific data aggregation to attain fine performance in terms of system life span, latency, and application seeming quality [9].

\section{PROPOSED WORK}

In the proposed work a technique named Energy Competent Steering Protocol Based on CSA (ECSPCSA) is projected to apply Cuckoo Search Algorithm (CSA) to the problem of electing the group leader $(\mathrm{CH})$ in the group set-up stage based on minimizing the intra group average space linking the member sensors and their relevant leaders $(\mathrm{CHs})$ and minimizing the total of fraction of remaining power/energy of active sensors in group to energy/power intensity of leader $(\mathrm{CH})$. It efficiently maximizes the arrangement life span and 
improves the constancy stage. The procedure of the ECSPCSA protocol is wrecked up into circles, where every circle starts with a set-up stage, when the Base Station (sink) locates leaders (CHs) and assigns associated sensors of every leader $(\mathrm{CH})$, chased by a steady-state stage, when the collected information is sent to leaders $(\mathrm{CHs})$ and receive in frames; then these frames are sent to the Base Station (sink).

The cluster heads are decided by sink using CSA out of the existing alive sensors nodes having residual energy more than a threshold energy level, and associated cluster members are established and thus groups are built as exposed in Figure 1. A threshold energy level is mean of energy intensity of all active sensors. Initially, the sink launches a small communication to wake up and to demand the Identifications, locations and power intensity and type of the node (advanced or normal) of every sensor in the sensor arrangement. Depending on the responded data from sensors, the sink utilizes CSA to elect $\mathrm{CHs}$ based on minimization of fitness function given by equation 3. The whole process to minimize the fitness function and cluster head selection is demonstrated in Figure 2. Also, the sink allocates the associated sensors of every leader $(\mathrm{CH})$ on the bases of minimum Euclidean distance. When group leaders $(\mathrm{CHs})$ are elected and associate members of leaders $(\mathrm{CH})$ are assigned, the sink (BS) launches a small communication to notify sensor network about leader $(\mathrm{CH})$ and associated members.

\section{Fitness Function:}

The problem of improving the clustering solution and to elect CHs can be invented as an optimization setback and exactly articulated as a combination of two functions as described below

$$
\mathrm{f} 1=\max _{\mathrm{i} \varepsilon\left(1, k_{o p t}\right)}\left\{\frac{\sum_{\forall n \in C_{i}} d(n, C H i)}{|C j|}\right\}
$$

where $k_{\text {opt }}$ symbolizes the $\mathrm{CHs}$ (leaders) count, $\mathrm{Ci}$ is the ith group renowned with group-head $\mathrm{CHi}$, and any member node, $\mathrm{n}$, belong to the group $\mathrm{Ci}$ that convinces the shortest space between $\mathrm{n}$ and $\mathrm{CHi}$. This function represents the highest of the sum of the Euclidean distance of associated sensors to their cluster heads $\mathrm{CHi}$ moreover $|\mathrm{C} j|$ is the count of sensors that belong to group $\mathrm{C}_{\mathrm{i}}$.

By reducing this function, it seems to reduce the intra-group average space between these member nodes and their individual leaders $(\mathrm{CHs}) .2^{\text {nd }}$ function is given by

$$
\mathrm{f} 2=\sum_{i=1}^{k_{o p t}}\left\{\frac{\sum_{\forall n \in C_{i}} E_{n}^{r e s}}{E_{C H i}^{r e s}}\right\}
$$

It represents the addition of fraction of remaining power of all sensors in a group to the power intensity of head of that cluster.

The objective (i.e. fitness) function is to minimize the following function

$$
\text { Fitness }=\alpha \times f 1+(1-\alpha) \times f 2
$$

By reducing the fitness function, it is projected that the group development and the leader $(\mathrm{CH})$ election of the Wireless Sensor Network can be optimized for growing the competence of energy expenditure within the arrangement.
The constant $\alpha$ denotes the donation of $f 1$ and $f 2$ in the fitness function. To avoid the election of the sensors having small energy intensity to be the leader $(\mathrm{CH})$, the $\mathrm{CH}$ sensors are elected from the group of applicants. Merely the sensors having energy intensity more than the mean power intensity of the entire sensors in the set-up can be the $\mathrm{CH}$ (leader) applicants.

\section{RESULT AND DISCUSSION}

To confine the performance of projected CSA in the network test instances and to study its actions against HSA algorithms, the simulations are carried out on various sensor node arrangements with different heterogeneous situations, every collected of 100 sensors arranged arbitrarily in a ground of $100 \mathrm{~m} \times 100 \mathrm{~m}$ sensor field. Experiments were performed considering $10 \%$ and $20 \%$ advanced nodes. BS (sink) is located at the midpoint of the field. The preliminary energy of a usual sensor is set to E0 $=0.5 \mathrm{~J}, \varepsilon \mathrm{fs}=10 \mathrm{pJ} / \mathrm{bit} / \mathrm{m} 2, \varepsilon \mathrm{mp}=$ $0.0013 \mathrm{pJ} / \mathrm{bit} / \mathrm{m} 4$, and EDA $=5 \mathrm{~nJ} / \mathrm{bit} /$ report. The message packet size that nodes transmit to their leaders $(\mathrm{CHs})$ as well as combined message packet size that a $\mathrm{CH}$ transmits to the base station is set to 4000 bits. Results for every simulation are given both quantitatively and qualitatively.

The discovery rate of alien eggs in CSA was fixed at 0.8 . The various parameters used in HSA were HMCR (harmony memory considering rate) and PAR (pitch adjustment rate), 0.95 and 0.7 correspondingly. The inhabitants' size was considered 20 and permitted to progress for 20 generations for both algorithms. And same fitness function was minimized using these algorithms to elect cluster head.

The records portray the amount of alive nodes against protocol rounds. Moreover, to provide a thorough insight into the performance of these algorithms, quantitative results are also incorporated summing up network life span (Tables 1 and 2 ) and the remaining energy in the network while protocol rounds continue (Tables 3 and 4). Make sure that in every table, the finest values are specified in bold.

The results in Tables 1 and 2 confirm the round count where a particular percentage of sensors expire for the compared algorithm. Outcomes in the tables evidently demonstrate the optimistic impact of the projected CSA for decreasing deceased nodes count while the algorithm rounds continue, and consequently, escalating the network life span. In Table 1, the increase in life span was $10.9 \%$ as compared with HSA, respectively. Also, in Table 2, the gain was $11.1 \%$ as compared with HSA correspondingly.

Supplementary comment can be made from the tables, which intimate the performance of CSA in opposition to HSA. CSA outperforms HSA in keeping the active sensors bigger than that of these algorithms throughout the network life time. This scrutiny can be quantitatively offered in Tables 1 and 2 for both types of WSNs.

Tables 3 and 4 demonstrate the optimistic impact of CSA for saving additional power in the arrangement at a chosen round space. Both clustering algorithms employ the whole energy. HSA disperses the network power sooner than other. And CSA preserves more energy than HSA algorithm in addition to longer stability period. 


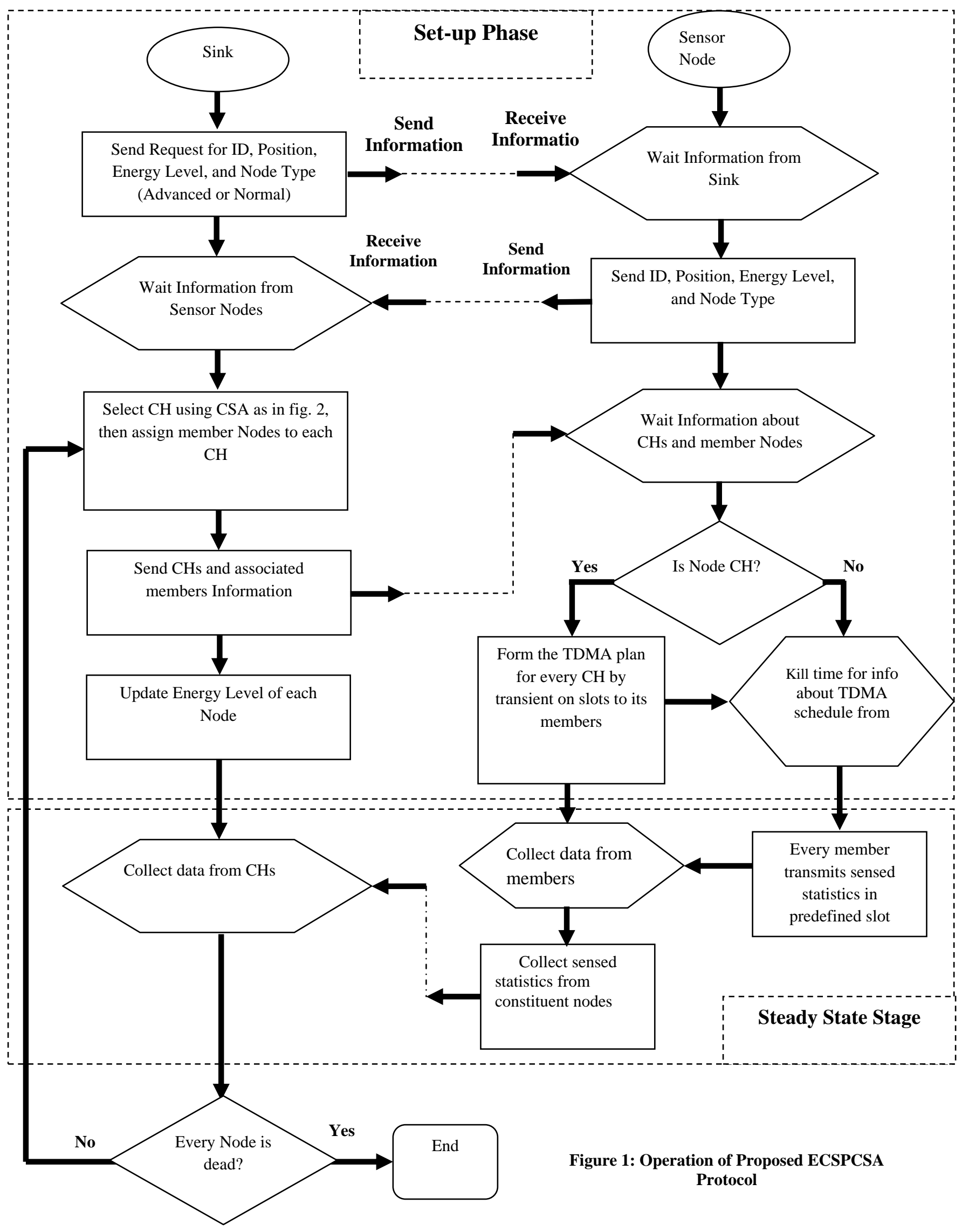




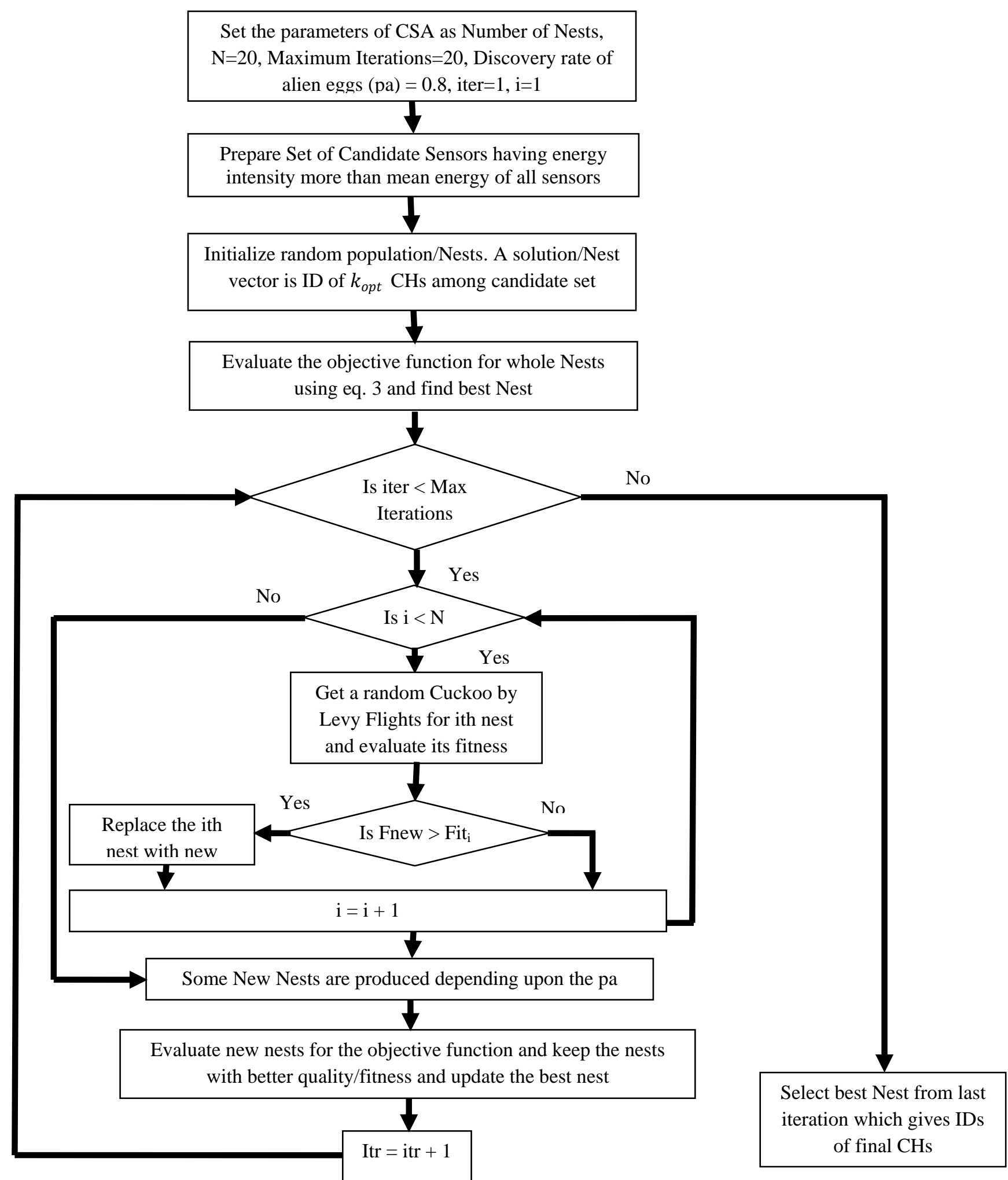

Figure 2: Flow chart of Leader $(\mathrm{CH})$ Election Algorithm using CSA in projected research 


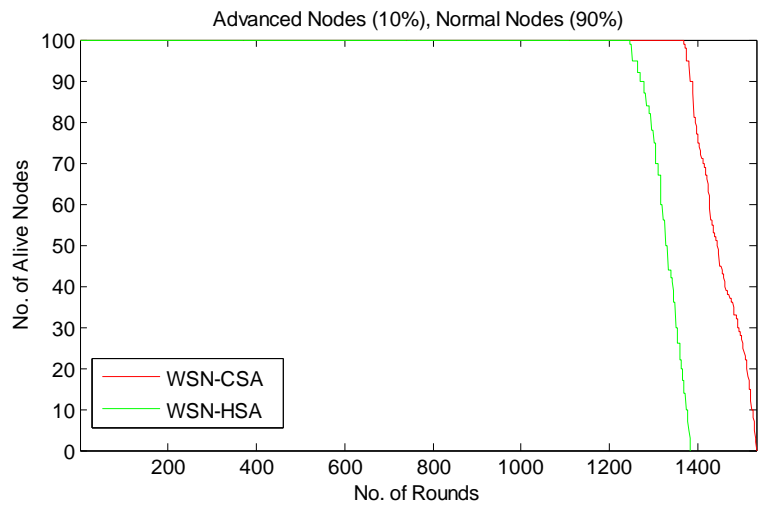

Figure 3: Active nodes count in the system versus rounds for $10 \%$ heterogeneity

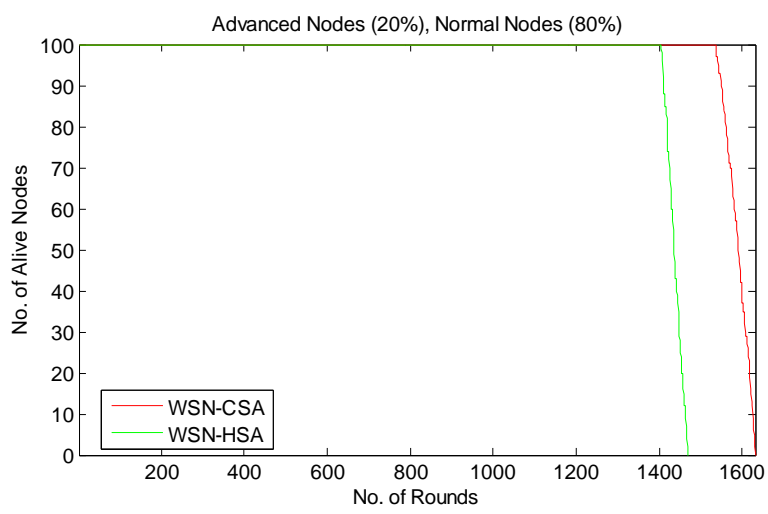

Figure 4: Active nodes count in the system versus rounds for $20 \%$ heterogeneity

Table 1: Percentage of dead nodes over rounds of WSNs (with $10 \%$ advanced nodes)

\begin{tabular}{|c|c|c|}
\hline \%dead nodes & HSA & CSA \\
\hline 10 & 1270 & $\mathbf{1 3 8 1}$ \\
\hline 20 & 1294 & $\mathbf{1 3 9 5}$ \\
\hline 30 & 1304 & $\mathbf{1 4 1 1}$ \\
\hline 40 & 1316 & $\mathbf{1 4 2 6}$ \\
\hline 50 & 1326 & $\mathbf{1 4 4 4}$ \\
\hline 60 & 1344 & $\mathbf{1 4 6 2}$ \\
\hline 70 & 1349 & $\mathbf{1 4 9 1}$ \\
\hline 80 & 1363 & $\mathbf{1 5 1 1}$ \\
\hline 90 & 1373 & $\mathbf{1 5 2 1}$ \\
\hline 100 & 1381 & $\mathbf{1 5 3 2}$ \\
\hline
\end{tabular}

Added remark can be worn from these tables, which reveal the actions of CSA next to HSA. CSA outperforms HSA by maintaining the alive nodes superior than that of these algorithms during the network life span. This study can be quantitatively accessed in Tables 1 and 2 for the two groups of WSNs.
Table 2: Percentage of dead nodes over rounds of WSNs (with $20 \%$ advanced nodes)

\begin{tabular}{|c|c|c|}
\hline \%dead nodes & HSA & CSA \\
\hline 10 & 1411 & $\mathbf{1 5 5 0}$ \\
\hline 20 & 1420 & $\mathbf{1 5 6 1}$ \\
\hline 30 & 1425 & $\mathbf{1 5 7 3}$ \\
\hline 40 & 1430 & $\mathbf{1 5 8 1}$ \\
\hline 50 & 1436 & $\mathbf{1 5 9 1}$ \\
\hline 60 & 1443 & $\mathbf{1 5 9 9}$ \\
\hline 70 & 1448 & $\mathbf{1 6 0 8}$ \\
\hline 80 & 1455 & $\mathbf{1 6 1 9}$ \\
\hline 90 & 1464 & $\mathbf{1 6 2 6}$ \\
\hline 100 & 1469 & $\mathbf{1 6 3 2}$ \\
\hline
\end{tabular}

Table 3: Residual energy over protocol rounds for a total of 1532 rounds (with $10 \%$ Heterogeneity)

\begin{tabular}{|c|c|c|}
\hline \%rounds & HSA & CSA \\
\hline 10 & 48.5207 & $\mathbf{4 8 . 5 4 6 9}$ \\
\hline 20 & 41.9187 & $\mathbf{4 1 . 9 4 7 6}$ \\
\hline 30 & 35.4784 & $\mathbf{3 5 . 5 1 8 5}$ \\
\hline 40 & 29.0916 & $\mathbf{2 9 . 1 4 3 9}$ \\
\hline 50 & 22.7007 & $\mathbf{2 2 . 7 5 6 6}$ \\
\hline 60 & 16.2976 & $\mathbf{1 6 . 3 6 8 7}$ \\
\hline 70 & 9.8917 & $\mathbf{9 . 9 7 9 4}$ \\
\hline 80 & $\mathbf{4 . 1 0 1 2}$ & 3.8098 \\
\hline 90 & 0.0580 & $\mathbf{1 . 3 5 5 8}$ \\
\hline 100 & $\wedge 0$ & $\mathbf{0}$ \\
\hline
\end{tabular}

Table 4: Residual energy over protocol rounds for a total of 1632 rounds (with $20 \%$ Heterogeneity).

\begin{tabular}{|c|c|c|}
\hline \%rounds & HSA & CSA \\
\hline 10 & 53.1779 & $\mathbf{5 3 . 1 9 4 0}$ \\
\hline 20 & 46.3223 & $\mathbf{4 6 . 3 2 4 8}$ \\
\hline 30 & 39.0908 & $\mathbf{3 9 . 1 3 2 9}$ \\
\hline 40 & 31.9665 & $\mathbf{3 2 . 0 7 5 2}$ \\
\hline 50 & 25.0922 & $\mathbf{2 5 . 2 4 0 0}$ \\
\hline 60 & 18.2558 & $\mathbf{1 8 . 4 3 4 8}$ \\
\hline 70 & 11.4311 & $\mathbf{1 1 . 6 3 2 6}$ \\
\hline 80 & 4.5673 & $\mathbf{5 . 8 1 8 1}$ \\
\hline 90 & $\wedge$ & $\mathbf{2 . 8 0 9 4}$ \\
\hline 100 & - & $\mathbf{0}$ \\
\hline
\end{tabular}




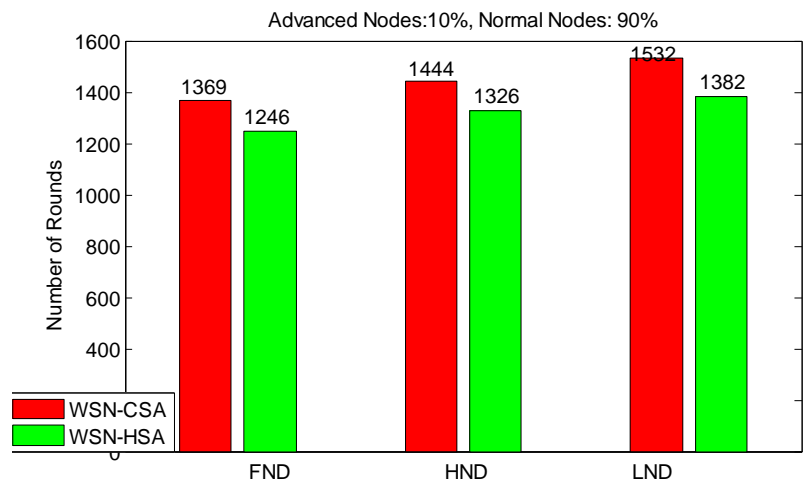

Figure 5: Quantity of rounds at LND, HND, FND (10\% Heterogeneity)

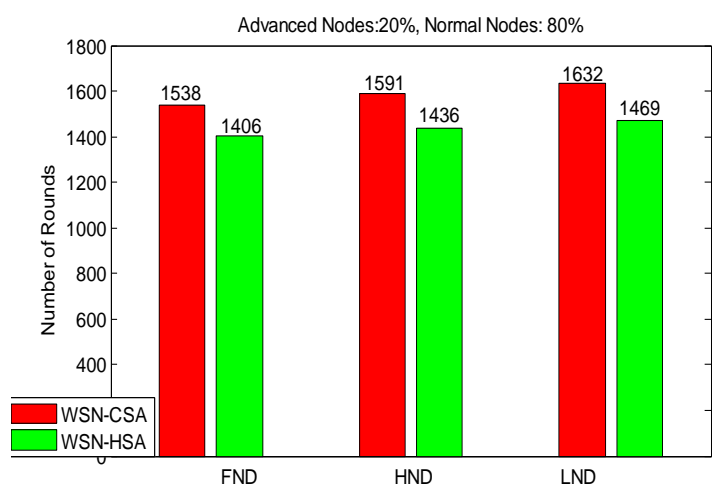

Figure 6: Quantity of rounds at LND, HND, FND (20\% Heterogeneity)

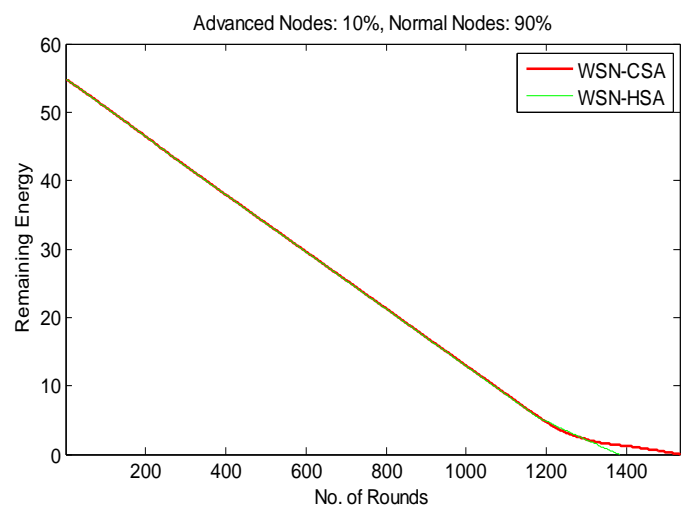

Figure 7: Residual energy of network with rounds (10\% Heterogeneity)

Fig 5 and Fig 6 shows the amount of rounds at FND, HND and LND for both the algorithms from these figures it can be observed that CSA algorithm in both cases performs better than the HAS algorithms in terms of stability period as well as network life span. The CSA algorithm extends the stability (Constancy phase) period by 123 in comparison to HSA in case of $10 \%$ heterogeneity. And it extends the stability period by 132 as compared to HSA in case of $20 \%$ heterogeneity.

Fig 7 and Fig 8 exhibit the residual energy of network as it proceeds towards final rounds and is very clear that remaining energy reduces with rounds but CSA algorithm outperforms the HSA by having more residual energy at any time of network for both the cases of heterogeneity.

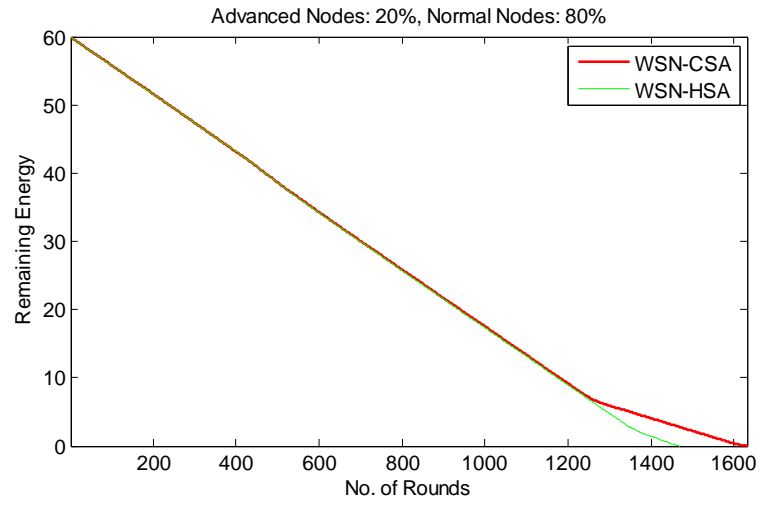

Figure 8: Residual energy of network with rounds (20\% Heterogeneity)

\section{CONCLUSION}

In the presented research, a new Artificial Bee Colony based Energy Efficient adaptive clustering hierarchy Protocol (ABCEEP) is proposed to proficiently lengthen the life span and stability period of WSNs. MATLAB simulation outcome illustrated that the proposed ABCEEP protocol is further energy proficient and added unswerving in clustering method in contrast to ERP (WSN-GA) as well as WSN with Harmony search algorithm for heterogeneous networks. The throughput of ABCEEP is always more than the previous algorithms so it can be said that more data is transferred in the network using the proposed technique in comparison to previous techniques with same or less amount of energy consumption. So Proposed routing protocol ABCEEP performs better almost all aspects of a WSN. As future work this CSA can be hybrid with some more optimization algorithms and can be applied to the same fitness function for cluster head election to get more survivable and stable period of WSN. The future work can also include mobility in the network and then check performance of ECSPCSA.

\section{REFERENCES}

[1] C. Kidd et al. The aware home: A living laboratory for ubiquitous computing research. In Proceedings of the Second International Workshop on Cooperative Buildings , 1999.

[2] L. Schwiebert, S. Gupta, and J. Weinmann. Research challenges in wireless networks of biomedical sensors. In Proceedings of the Seventh Annual International Conference on Mobile Computing and Networking, 2001.

[3] A. Mainwaring, J. Polastre, R. Szewczyk, D. Culler, and J. Anderson. Wireless sensor networks for habitat monitoring. In Proceedings of the ACM International Workshop on Wireless Sensor Networks and Applications, 2002.

[4] R. Parvin and Vasanthanayaki,"Particle Swarm Optimization based Clustering by Preventing Residual Nodes in Wireless Sensor Networks", IEEE, pp. 1-11, 2015.

[5] D. C. Hoang , P. Yadav, R. Kumar and S. K. Panda, "Real-Time Implementation of a Harmony Search Algorithm-Based Clustering Protocol for EnergyEfficient Wireless Sensor Networks", IEEE Transactions On Industrial Informatics, Vol. 10, No. 1, February 2014. 
[6] B. A. Attea and E. A. Khalil, “A new evolutionary based routing protocol for clustered heterogeneous wireless sensor networks", ELSEVIER, pp. 1950-1957, 2012.

[7] A. Zahmatkesh and M. H. Yaghmaee,“ A Genetic Algorithm-Based Approach for Energy- Efficient Clustering of Wireless Sensor Networks", International Journal of Information and Electronics Engineering, Vol. 2, Issue No. 2, 2012.

[8] G. Smaragdakis, I. Matta, A. Bestavros, SEP: a stable election protocol for clustered heterogeneous wireless sensor networks, in: Second International Workshop on
Sensor and Actor Network Protocols and Applications (SANPA 2004), Boston, MA, 2004.

[9] W. R. Heinzelman, A. Chandrakasan, and H. Balakrishnan," An Application-Specific Protocol Architecture for Wireless Microsensor Networks", Volume-1, Issue No. 4, pp. 660-670, 2002.

[10] W. R. Heinzelman, A. Chandrakasan, and H. Balakrishnan, "Energy-Efficient Communication Protocol forWireless Microsensor Networks", Proceedings of the 33rd Hawaii International Conference on System Sciences, pp. 1-10, 2000. 